\title{
Improved Surgical Site Infection (SSI) rate through accurately assessed surgical wounds
}

\author{
Honeymol John, Abdelrahman Nimeri, SAMER ELLAHHAM
}

Sheikh Khalifa Medical City (SKMC), United Arab Emirates

\begin{abstract}
Sheikh Khalifa Medical City's (SKMC) Surgery Institute was identified as a high outlier in Surgical Site Infections (SSI) based on the American College of Surgeons National Surgical Quality Improvement Program (ACS NSQIP) - Semi-Annual Report (SAR) in January 2012. The aim of this project was to improve SSI rates through accurate wound classification.

We identified SSI rate reduction as a performance improvement and safety priority at SKMC, a tertiary referral center. We used the American College of Surgeons National Surgical Quality Improvement Program (ACS NSQIP) best practice guidelines as a guide. ACS NSQIP is a clinical registry that provides risk-adjusted clinical outcome reports every six months. The rates of SSI are reported in an observed/expected ratio. The expected ratio is calculated based on the risk factors of the patients which include wound classification.

We established a multidisciplinary SSI taskforce. The members of the SSI taskforce included the ACS NSQIP team members, quality, surgeons, nurses, infection control, IT, pharmacy, microbiology, and it was chaired by a colorectal surgeon. The taskforce focused on five areas: pre-op showering and hair removal, skin antisepsis, prophylactic antibiotics, peri-operative maintenance of glycaemia, and normothermia. We planned audits to evaluate our wound classification and our SSI rates based on the SAR.
\end{abstract}

Our expected SSI rates in general surgery and the whole department were $2.52 \%$ and $1.70 \%$ respectively, while our observed SSI rates were $4.68 \%$ and $3.57 \%$ respectively, giving us a high outlier status with an odd's ratio of 1.72 and 2.03 . Wound classifications were identified as an area of concern. For example, wound classifications were preoperatively selected based on the default wound classification of the booked procedure in the Electronic Medical Record (EMR) which led to under classifying wounds in many occasions.

A total of 998 cases were reviewed, our rate of incorrect wound classification assignment was $36 \%$, and the worst rates were in appendectomies (97\%). Over time our incorrect wound classification decreased down to $22 \%$, while at the same time our actual SSI wound occurrences per month and our odds ratio of SSI in the department have decreased an average of six to three per month.

We followed the best practice guidelines of the ACS NSQIP. Accurate assessment of wound classification is necessary to make sure the expected SSI rates are not falsely high if wounds are under-classified. The present study shows that accurate wound classification in contaminated and dirty wounds can lead to lower odds ratio of SSI.

\section{Problem}

Sheikh Khalifa Medical City's Surgery Institute (Abu Dhabi, UAE) participates in the American College of Surgeons National Surgical Quality Improvement Program (ACS NSQIP) since August of 2009. We were identified as a high outlier in SSI in general, vascular surgery and the entire Surgery Institute. Our problem of SSI appeared to be a systemic problem rather than a problem of a specific division. We were identified as a high outlier in SSI based on the Semi-Annual Report in January 2012.

The SSI taskforce recommended that we conduct a retrospective chart review of all SSI cases in 2013 detected by the ACS NSQIP to better understand the SSI problem and detect any patterns. We discovered that the accuracy of wound classification was lost when the hospital migrated to the Electronic Medical Record (EMR) Cerner. Once the hospital migrated to Cerner, the default wound classification in the system for different procedures was used automatically. Unless the nursing staff actively changed the wound classification, this default classification was entered automatically. It appeared that no health care professionals have reviewed these default wound classifications. The wound classification is important because the ACS NSQIP reports the odds ratio of SSI by calculating the Observed/Expected ratio (O/E ratio) based on the risk factors of the patient. One of the most important risk factors in SSI is wound classification.

The expected SSI rate is calculated by evaluating the risk factors of the patient. Other factors contributing to SSI include duration of surgical scrub, maintenance of body temperature, the use of skin antisepsis, preoperative shaving, duration of the operation, and antimicrobial prophylaxis. This was in addition to ventilation of the operating room, inadequate sterilization of instruments, the presence of foreign material at the surgical site, surgical drains, and surgical technique. Poor surgical technique includes poor hemostasis, failure to obliterate dead space, and tissue trauma. 


\section{Background}

The impact of SSI on morbidity, mortality, and cost of care were identified as a priority. Based on Centers for Disease Control and Prevention (CDC) National Nosocomial Infections Surveillance (NNIS) system reports, SSIs are the third most frequently reported nosocomial infection, accounting for $14 \%$ to $16 \%$ of all nosocomial infections among hospitalized patients.

Among surgical patients, however, SSIs are the most common nosocomial infection, observed in $38 \%$ of cases. Two-thirds of these infections are due to the incision, whereas one-third is due to infection of the organs or spaces during surgery.

According to CDC, a surgical site infection is an infection that occurs after surgery in the part of the body where the surgery took place. It can be superficial, involving the skin only or more serious involving tissues under the skin, organs, or implanted material. The symptoms are: (a) redness and pain around the area where surgery has been done (b) oozing of cloudy fluid from the surgical wound (c) fever.

Surgical site infections are categorized into four classes depending on wound type. (a) Class 1 is a clean wound, (b) Class 2 is a cleancontaminated wound, (c) Class 3 is a contaminated Wound, and (d) Class 4 is a dirty-infected wound.

Patients with SSI are more likely to remain in the hospital for a longer duration, tend to become more serious, and have an increased incidence of morbidity, costs, and mortality.

\section{Baseline measurement}

Our expected SSI rates in general surgery and the whole department were $2.52 \%$ and $1.70 \%$ while our observed SSI rates were $4.68 \%$ and $3.57 \%$, giving us a high outlier status with an odd's ratio of 1.72 and 2.03. Wound classifications had been identified as an area of concern since the start of Cerner documentation.

For example, wound classifications were preoperatively selected based on the default wound classification of the booked procedure in Cerner, leading to under-classifying wounds on many occasions.

A total of 998 cases were reviewed; our rate of incorrect wound classification assignment was $36 \%$, and the worst rates were in appendectomies (97\%). Over time our incorrect wound classification decreased down to $22 \%$, and at the same time our actual SSI wound occurrences per month in the department have decreased from an average of six to three per month.

See supplementary file: ds4733.docx - "Table \& Graph showing decrease in incorrect wound classification from 2013 to 2014 \& the Odd's ratio"

\section{Design}

As a result, a multidisciplinary team, SSI taskforce was put together to address the surgical site infection rates relevant to general, vascular surgery and the entire Surgery Institute. A root-cause analysis was done and multiple interventions were adopted including the adaptation of the Surgical Site Infection bundle care approach, the implementation of the WHO Surgical Safety Checklist of 2010 , as well as staff education and patient education.

Post-operated patients' charts were retrospectively analyzed and we found that inappropriate wound classification was the main contributory risk factor responsible for our high observed/expected SSI rates in general, vascular surgery, and the entire surgery Institute. In addition, the taskforce implemented five key recommendations to improve our SSI rates, as follows: pre-op showering and hair removal, skin antisepsis, prophylactic antibiotics, perioperative maintenance of glycaemia and normothermia. We also implemented the best-practice systems and processes using education and modifications in the Cerner system.

The baseline period for measuring SSI rates was calendar year 2012 through 2014. For this project, all surgeries (excluding cardiac, transplant, trauma, pediatric surgery) were included in the audit. The patients' charts were referred to see the nurse notes and wound classification marked. The following wound classification criteria were followed:

Patients were stratified by wound classification (clean, clean/contaminated, contaminated, and dirty) as defined by ACSNSQIP. Surgical site infection was the primary outcome of the study.

Review of patient files, post-operative disclosed that one of the reasons for our increase in SSI rates was improper wound classification. The goal was to reduce our SSI rates in general, vascular surgery, and the entire surgery institute.

\section{Strategy}

Our SSI taskforce made several recommendations as: Patients will be asked to take a shower with chlorhexidine prior to surgery; we will only use electric clippers to shave the hair and this will not happen in the operating room (as we did in our previous practice) but in the preoperative admission area; an alcohol based prep will be used in the operating room; and in the operating room, only nurses will be allowed to prep the surgery site.

In addition, the competency of skin prepping was reviewed for all the nurses.

A specific lactic antibiotic was chosen for each surgical division after discussion with microbiology pharmacy and the concerned surgeons. Furthermore, it was expressed to Anesthesia that they will have to give the antibiotics prior to induction and re-dose the antibiotics if necessary, and that the surgeons were made responsible for stopping the antibiotics less than 24 hours from surgery and continue temperature monitoring in recovery room for all patients.

We followed the best practice guidelines of the ACS NSQIP. In 
BMJ Quality Improvement Reports

addition (because the risk adjustment for patients is done based on the perioperative risk factors) we felt that accurate wound classification is important in assessing our patients risk for SSI appropriately. In regards to wound classification, this meant that all default wound classifications were reviewed in the EMR Cerner. In addition, we started auditing the accuracy of wound classification assignment by the nursing staff. Furthermore, we added a mandatory pop-up window on the nursing documentation menu for the operating room nurses. This pop-up window asked the nurse, the comment and click affirmatively that the accuracy of the wound classification was discussed with the surgeon.

\section{Results}

SSI rates were 1.84 (1.24-2.45) in 2010, a statistically significant high outlier. SSI rates were 2.07 (1.44-2.69) in 2013 statistically significant high outlier. However, SSI rates came down to 1.11 (0.65-1.62) (Expected in odds ratio). The odds ratio decreased from previous $1.72 \& 2.03$ to 1.45

We followed the best practice guidelines of the ACS NSQIP.

See supplementary file: ds4969.docx - "Result Report \& Guidelines"

\section{Lessons and limitations}

General, vascular, and the entire surgery institute, expected SSI rates were accurately assessed by improving our wound classification accuracy. In addition, our SSI rates were reduced and controlled after implementing the following:

- Nursing education (skin prep competency) : (a) Hair removal exclusively at the pre-operative department and (b) Pre-operative showering for all patients implemented as a standard practice

- Default classification for appendectomies changed from "clean contaminated" to "contaminated"

- OR nurses asked to check wound class with surgeon at end of each case

- Mandatory check box to verify wound class added to Cerner.

These activities demonstrated the lessons learned from the process implementation, outcome successes, and the cross-institutional learning about SSI prevention, facilitating the goal of sharing of the successes in implementation of improvement plans to reduce SSIs.

\section{Conclusion}

We followed the best practice guidelines of the ACS NSQIP. Accurate assessment of wound classification is an important factor in delivering expected SSI rates. Failing to do so can lead to falsely high O/E SSI rates.

These interventions are bundled together and considered integral components of the best practices care we could provide to our patients. The study also provided an opportunity for sharing feedback on appropriate data with healthcare providers.

\section{References}

1. ACS NSQIP Newsletter https://site.acsnsqip.org/newsletter/reducing-ssi-rates

2. Mangram AJ et al. Infect Control Hosp Epidemiol 1999; 20:250-278.

3. Berríos-Torres, MD, Surgical Site Infection (SSI) Toolkit Activity, Centers for Disease Control and Prevention, infect Control Hosp Epidemiol 2008;29:S51-S61, Prevention of surgical site infection, 1999. Available at www.cdc.gov/hai/pdfs/toolkits/SSI_toolkit021710SIBT_revis ed.pdf, www.cdc.gov/hai/ssi/ssi.html

4. Alicia J Mangram MD et al. Guideline for Prevention of Surgical Site Infection - I. Surgical Site Infection (SSI): An Overview. Infect Contr Hosp Epidemiol 1999. 20(4):247-28. Available at http://www.cdc.gov/hicpac/SSI/002 SSI.html

5. Guideline for Prevention of Surgical Site Infection, 1999, Table 7 Surgical Wound Classification. Available at: http://www.cdc.gov/hicpac/SSI/table7-8-9-10-SSI.html

6. Angela M Ingraham, Mira Shiloach, E Patchen Dellinger, et.al. ACS NSQIP Best Practices Guidelines, Prevention of Surgical Site Infections, July 1999.

7. Suzanne M. Pear, RN, Ph.D, CIC, Patient Risk Factors and Best Practices for Surgical Site Infection Prevention.

Available at www.halyardhealth.com/.../patient_risk_factors_best_practic es_ssi.pdf

\section{Declaration of interests}

Nothing to declare.

\section{Acknowledgements}

Co-authors:

Dr. Abdelrahman Nimeri, Chief, Division of General, Thoracic and Vascular Surgery, SKMC. Director of Bariatric \& Metabolic Institute (BMI) Abu Dhabi.

Dr. Samer Ellahham, MD, Chief Quality Officer, SKMC.

Acknowledgements:

Ruby Philip, RN SCR, ACS-NSQIP, Surgical Institute, SKMC.

Karen McKenna, RN, Surgical Institute, SKMC.

Dr. Nicolas P. Turrin, Senior Risk Officer, Quality Management, SKMC. 